\title{
Intact subliminal processing and delayed conscious access in multiple sclerosis
}

\author{
Françoise Reuter ${ }^{\mathrm{a}, \mathrm{b}}$, Antoine Del Cul ${ }^{\mathrm{c}}$, Bertrand Audoin ${ }^{\mathrm{a}, \mathrm{b}, *}$, Irina Malikova ${ }^{\mathrm{a}, \mathrm{b}}$, \\ Lionel Naccache ${ }^{\mathrm{c}, \mathrm{d}}$, Jean Philippe Ranjeva ${ }^{\mathrm{a}}$, Olivier Lyon-Caen ${ }^{\mathrm{d}}$, André Ali Chérif ${ }^{\mathrm{b}}$, \\ Laurent Cohen ${ }^{\mathrm{c}, \mathrm{d}}$, Stanislas Dehaene ${ }^{\mathrm{c}, \mathrm{e}}$, Jean Pelletier ${ }^{\mathrm{a}, \mathrm{b}}$ \\ ${ }^{a}$ Centre de Résonance Magnétique Biologique et Médicale (CRMBM) UMR CNRS 6612, Faculté de Médecine, \\ Université de la Méditerranée, Marseille, France \\ ${ }^{\mathrm{b}}$ Department of Neurology, Timone University Hospital, Marseille, France \\ ' INSERM, CEA, Cognitive Neuroimaging Unit, Service Hospitalier Frédéric Joliot, Orsay, France \\ ${ }^{\mathrm{d}}$ Department of Neurology, Salpêtrière Hospital, Paris, France \\ ${ }^{\mathrm{e}}$ Collège de France, Paris, France
}

Received 4 September 2006; received in revised form 2 March 2007; accepted 4 April 2007

Available online 19 April 2007

\begin{abstract}
Periventricular white matter damage affecting large bundles connecting distant cortical areas may constitute the main neuronal mechanism for the deficit of controlled information processing observed in patients with early multiple sclerosis (MS). Visual backward masking has been demonstrated to affect late stages of conscious perception involving long-range interactions between visual perceptual areas and higher level integrative cortices while leaving intact early feed-forward visual processing and even complex processing such as object recognition or semantic processing. We therefore hypothesized that patients with early MS would have an elevated masking threshold, because of an impairment of conscious perception whereas subliminal processing of masked stimuli would be preserved.

Twenty-two patients with early MS and 22 normal controls performed two backward-masking experiments. We used Arabic digits as stimuli and varied quasi-continuously the temporal interval with a subsequent mask, thus allowing us to progressively "unmask" the stimuli. We finely quantified the visibility of the masked stimuli using both objective and subjective measures, thus obtaining accurate estimates of the threshold duration for access to consciousness. We also studied the priming effect caused by the variably masked numbers on a comparison task performed on a subsequently presented and highly visible target number.

The threshold for access to consciousness of masked stimuli was elevated in MS patients compared to controls, whereas non-conscious processing of these stimuli, as measured by priming, was preserved. These findings suggest that conscious access to masked stimuli depends on the integrity of large-scale cortical integrative processes, which involve long-distance white matter projections, and are impaired due to diffuse demyelinating injury in patients with early MS.
\end{abstract}

(C) 2007 Elsevier Ltd. All rights reserved.

Keywords: Multiple sclerosis; Priming; "Workspace" theory; Consciousness; Cognitive impairment; Large scale network

\section{Introduction}

Cognitive impairment is a common finding in multiple sclerosis (MS), an inflammatory demyelinating disease of the human central nervous system (Heaton, Nelson, Thompson, Burks, \&

\footnotetext{
* Corresponding author at: Service de neurologie, CHU Timone, 265 Boulevard Saint Pierre, 13005 Marseille, France.

E-mail address: Bertrand.audoin@ap-hm.fr (B. Audoin).
}

Franklin, 1985; Peyser, Edwards, Poser, \& Filskov, 1980; Rao, Leo, Bernardin, \& Unverzagt, 1991). At the early stage of the disease, cognitive dysfunction is mainly focused on integrative processes requiring consciousness such as working memory, speed of information processing, attention and executive function (Callanan, Logsdail, Ron, \& Warrington, 1989; Deloire et al., 2005; Feuillet et al., 2007). The conscious neuronal workspace theory (Dehaene, Kerszberg, \& Changeux, 1998; Dehaene, Sergent, \& Changeux, 2003) emphasizes the role of distributed neurons with long-distance connections, particularly 
dense in prefrontal, cingulate, and parietal regions, which are capable of interconnecting multiple specialized processors that have to interact synchronously during conscious controlled processing. According to this theory, the integrity of the white matter may be one main prerequisite for performing these high-level cognitive tasks involving several distant brain areas that have to interact rapidly. We infer that disruption of connectivity inside large-scale cortical networks, occurring as a consequence of diffuse white matter damage, may constitute the main neuronal mechanism of the deficit in controlled information processing in patients at the early stage of MS (Au Duong, Audoin et al., 2005; Au Duong, Boulanouar et al., 2005; Audoin et al., 2005; Deloire et al., 2005). Even if diffuse white matter injury may also alter or slow the conduction of information to primary visual and other specialized processing areas, one may expect encapsulated processes, supported primarily by short- and medium-range neuronal networks to have a greater probability of being preserved than higher level controlled processes, at least at the early stage of MS when no significant neuronal loss has been demonstrated (Audoin et al., 2007; Fernando et al., 2004; Ranjeva et al., 2003).

Here we tested this idea using a backward visual masking paradigm, adapted from metacontrast masking (Breitmeyer \& Ogmen, 2000), which consists in suppressing the visibility of a briefly presented stimulus by a mask presented shortly after. Backward masking seems particularly relevant to explore conscious integrative processes, as it provides a simple and highly reproducible situation in which the threshold for conscious access can be measured (Delcul, Dehaene, \& Leboyer, 2006).

Breitmeyer and colleagues initially proposed that masking depends primarily on the interactions between transient (magnocellular) and sustained (parvocellular) channels within the early visual pathways (Breitmeyer \& Ogmen, 2000). More recent models do not deny that masking may occur at many stages in the visual system, but argue that the lack of conscious perception is ultimately linked to disruption of late stages of cortico-cortical integration between bottom-up inputs and a top-down attentional signal (Dehaene \& Changeux, 2004; Dehaene \& Naccache, 2001; Dehaene et al., 1998, 2003; Delcul et al., 2006; Di Lollo, Enns, \& Rensink, 2000; Lamme, 2003). In those models, a distinction is introduced between two main stages of processing (Dehaene, Changeux, Naccache, Sackur, \& Sergent, 2006; Lamme \& Roelfsema, 2000). During a first stage corresponding to non-conscious visual processing, the visual stimulus is processed by a series of brain areas activated in a bottom-up manner. In a second stage, top-down feedback from higher areas to lower-level sensory regions establishes a self-amplified reverberant neuronal assembly which connects together distant brain areas, and is associated with conscious reportability. This reverberation is thought to occur via multiple cortico-cortical loops, first locally within the visual areas, then increasingly globally with more distant prefrontal and parietal regions.

According to such models, one masked stimulus can fail to reach consciousness if the mask replaces the stimulus before this recurrent activity has become stable. In support of these views, Haynes et al. (Haynes, Driver, \& Rees, 2005), using a metacontrast masking paradigm, found that conscious perception was correlated to activity in both striate and extrastriate visual cortex (area V5, fusiform gyrus) as well as to parieto-frontal areas. Furthermore, conscious perception was associated with increased functional connectivity between primary visual cortex and multiple visual areas including the fusiform gyrus. Many other studies have observed a failure of propagation of activity into higher level visual and parieto-frontal cortices, both during visual masking (Lau \& Passingham, 2006) as well as in related conditions such as the attentional blink (Beck, Rees, Frith, \& Lavie, 2001; Marois, Chun, \& Gore, 2000; Marois, Yi, \& Chun, 2004; Sergent, Baillet, \& Dehaene, 2005).

Specific theories differ as to whether relatively medium-range loops within occipito-temporal cortices are sufficient to create a conscious percept (Lamme, 2006) or whether a more global brain-scale access is needed, involving distant parietal (Di Lollo et al., 2000) or fronto-parietal areas (Dehaene et al., 2006). However, here we shall not be concerned with this distinction, but solely with the notion that recurrent activity involving mediumto long-range interactions between visual perceptual areas and higher level fronto-parietal cortices is associated with conscious perception during masking, as opposed to the predominantly local and feedforward short-range or "horizontal" interactions inside visual areas encountered during non-conscious visual processing. In early MS, the higher susceptibility to pathological processes of large periventricular white matter bundles compared to local white matter connections suggests a preferential impairment of long-range interactions (Au Duong, Audoin et al., 2005; Au Duong, Boulanouar et al., 2005; Cader, Cifelli, Abu-Omar, Palace, \& Matthews, 2006). Accordingly, early MS patients should have abnormal conscious access for masked stimuli and relatively preserved non-conscious processing.

The aim of the present study is to test the hypothesis that (1) patients with MS have a delayed access to consciousness for masked stimuli in comparison to healthy subjects, secondary to long distance cortico-cortical connections injury, and (2) subliminal processing of these stimuli remains normal or only slightly impaired in comparison to the large deficit in conscious access. In order to test this hypothesis, we used a visual backward masking task previously described in a recent study of patients with schizophrenia (Delcul et al., 2006), a disease that may also involve abnormal long-range connectivity. This masking paradigm allows us to compare conscious perception and non-conscious perception of visual stimuli between patients and healthy controls. This task includes Arabic digits as stimuli, and uses a quasi-continuous variation of the interval between the digit and the subsequent mask, allowing us to progressively "unmask" the stimulus. Using this task, we studied both the subliminal priming effect caused by these variably masked numbers, as well as their degree of visibility, to precisely quantify the threshold duration for access to consciousness in a group of early relapsing remitting MS patients compared to matched healthy controls.

\section{Method}

\subsection{Subjects}

A group of 22 patients with early relapsing remitting multiple sclerosis (mean disease duration 28 months, mean age 30.7 years, range $16-49,17$ women and 5 
Table 1

Demographic, clinical and neuropsychological characteristics of subjects

\begin{tabular}{llll}
\hline & MS patients & Normal controls & $P$ value \\
\hline Age mean (S.D.) & $30.7( \pm 8.9)$ & $26.8( \pm 8.5)$ & 0.115 \\
Education mean (S.D.) & $12.6( \pm 3.2)$ & $14.5( \pm 2.8)$ & 0.067 \\
$\begin{array}{l}\text { Disease duration } \\
\quad \text { (months) mean (S.D.) }\end{array}$ & $28( \pm 21)$ & & \\
EDSS median (range) & $1(0-2)$ & & \\
EDSS mean (S.D.) & $0.9( \pm 0.8)$ & & \\
MFIS mean (S.D.) & $27.5( \pm 21)$ & $9.6( \pm 9.2)$ & 0.001 \\
$\quad \begin{array}{l}\text { Word list generation } \\
\quad \text { mean (S.D.) }\end{array}$ & $29( \pm 10)$ & $39( \pm 9.8)$ & 0.004 \\
$\quad$ SDMT mean (S.D.) & $50( \pm 7.8)$ & $57.5( \pm 8.4)$ & 0.006 \\
\hline
\end{tabular}

men) participated in the study. A control group of 22 age-, sex- and educational level-matched healthy subjects has been also included. All were right-handed and native French speakers (Table 1).

Exclusion criteria included alcohol or other drug abuse, optic neuritis history, impaired visual acuity and the existence of scotoma on the visual field. All patients fulfilled multiple sclerosis diagnosis according to McDonald's criteria. Seven patients were treated with disease modifying drugs (beta interferon). None of the patients had experienced a relapse or treatment with steroids in the preceding 3 months. Disability was assessed with the Kurtzke expanded disability status scale (EDSS) (Kurtzke, 1983).

Minimal neuropsychological assessment was performed using the brief repeatable battery (BRB) (Rao, 1990). Fatigue was evaluated using the Modified Fatigue Impact Scale (Fisk et al., 1994).

\subsection{Visual backward masking task (Delcul et al., 2006)}

The test was delivered through a PC laptop using the E-Prime software. Response times were recorded with millisecond accuracy using a dedicated response box (Psychology Software Tools, Inc.).

In our visual masking paradigm (Delcul et al., 2006), stimuli presentation began with central cross fixation. Then, a first number (color: black, Arabic number; font: courier new; size: 36; backcolor: white) that we will call the "prime" appeared. It was followed, after a variable delay, by a masking shape surrounding the prime position without spatial overlap. This mask contained in its structure a second number, the "target" (color: black, Arabic number; font: courier new; size: 36; backcolor: white), and 3 letters (M, Z, Z) (color; black; font: courier new; size: 36; backcolor: white) (see Fig. 1). The prime was presented for $16 \mathrm{~ms}$ and the target for $250 \mathrm{~ms}$. The prime was positioned randomly in one of the four positions at the square tops (1.4 visual angle) relative to the central fixation cross (upper left, upper right, bottom left, bottom right). Visual angle of the target was $1^{\circ}$ relative to the central fixation cross. We varied the interval between the prime and the subsequent mask, thus allowing us to progressively unmask the stimulus digit. The delay between the onset of the prime and the onset of the mask could take one out of eight values $(0,16,33$, $50,66,83,100$ and $150 \mathrm{~ms}$ ), referred as the stimulus onset asynchrony (SOA). For the SOA value of $0 \mathrm{~ms}$, the prime and the mask had the same onset, but the target persisted after the prime had disappeared.

As regards to the strict definition of metacontrast masking (Breitmeyer \& Ogmen, 2000) where (1) the mask does not overlap spatially with the stimulus, (2) the contours/edges of the mask and the stimulus typically overlap, and (3) the masking function is U-shaped, our experiment fulfilled the first condition and partially the second. Absence of U-shaped masking function (Delcul et al., 2006) may be related to the long duration of the mask, which makes our masking paradigm similar to four-dot substitution masking (Di Lollo et al., 2000).

The stimulus set consisted of 16 pairs of prime and target numbers, consisting in all pairs of the numbers 1, 4, 6 and 9 written in Arabic format. As a consequence, the following factors could be analyzed: response congruity (whether or not the prime and target fell on the same side of 5), and repetition (within the congruent trials, whether or not the prime and target were the same number). Then, trials could be divided in three conditions defined by the prime-target relation: congruent repeated, congruent non-repeated and incongruent.

The experiment began with an explanation of the task and a visualization of examples. Two experiments were then performed in a fixed order.

\subsubsection{Priming experiment}

In the first experiment referred to as the "priming experiment", subjects were asked to compare each target number with 5, pressing the right-hand key as fast as possible for numbers larger than 5 and the left hand key for numbers smaller than 5. This experiment consisted in 320 experimental trials ( 8 blocks of 40 trials, one block for each delay). The different delays were blocked in order to facilitate the subject's task; it was felt that, if the delays had been mixed, it would have been too difficult for patients to avoid responding to the prime on

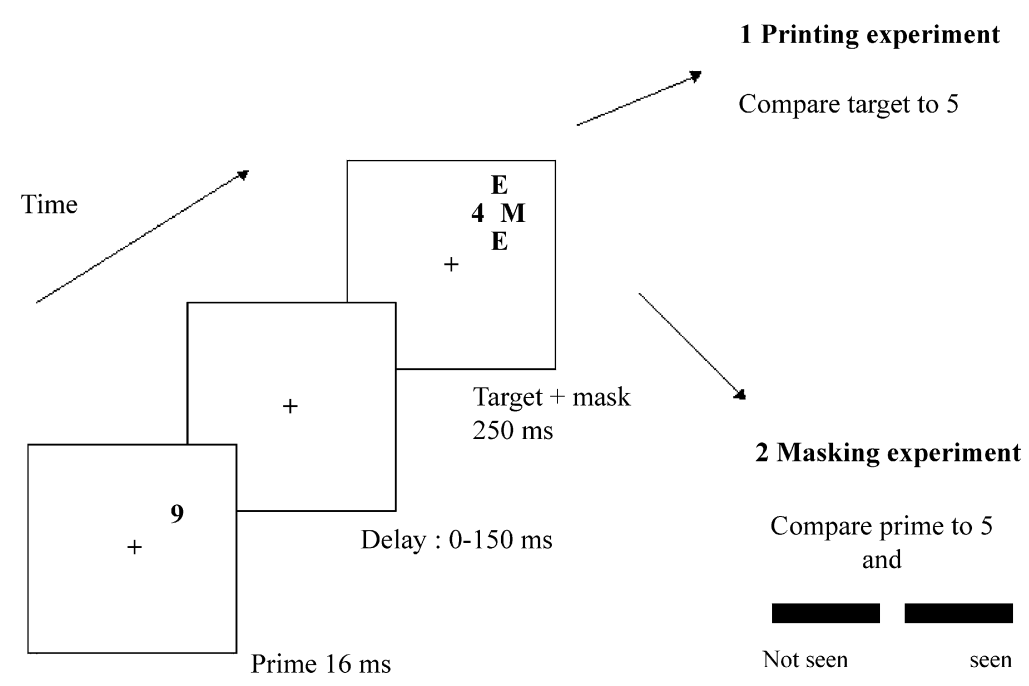

Fig. 1. Experiment design. The prime was presented for $16 \mathrm{~ms}$ at one of four positions (1.4 ${ }^{\circ}$ above or below and $1.4^{\circ}$ right or left to the fixation cross). The mask (duration of presentation $250 \mathrm{~ms}$ ) was composed of three letters (E, M, E) and the target number ( $1^{\circ}$ from the fixation cross). Those four symbols surrounded the location of the previous prime number without touching it. In the first experiment, referred to as the "priming experiment", subjects were asked to compare each target number with 5, pressing the right-hand key as fast as possible for numbers larger than 5 and the left-hand key for numbers smaller than 5 . The second experiment aimed at measuring the consciousness threshold in two different ways. We measured an objective visibility threshold by examining subjects' ability to perform the number comparison task on the prime. We also measured a subjective threshold by collecting introspective ratings of prime visibility, on a subjective continuous scale. 
conscious trials. Blocking helped them to learn to focus on the target and neglect the prime, regardless of its visibility.

\subsubsection{Masking experiment}

The second experiment aimed at measuring the threshold for access to consciousness. We measured an objective visibility threshold by examining subjects' ability to perform a number comparison task on the prime. We also measured a subjective threshold by collecting introspective ratings of prime visibility. Subjects were asked to move a cursor on a continuous scale from "seen" to "not seen" (Delcul et al., 2006; Sergent \& Dehaene, 2004). This scale was not graduated but was divided by the computer into 21 positions $(0-20)$. The experiment consisted in 20 trials for each delay, for a total 180 randomly presented trials.

In order to characterize, for each subject, an objective and a subjective threshold for access to consciousness, we used non-linear regression to fit the data with a sigmoid defined as $f(x)=\alpha_{1}+\left(\alpha_{2} /\left(1+\mathrm{e}^{-\alpha_{3}\left(x-\alpha_{4}\right)}\right)\right)$ where the $\alpha_{i}$ are free parameters.

\section{Results}

\subsection{Clinical and neuropsychological characteristics}

All data are displayed in Table 1. Significant between-groups differences were found in Word List Generation task $(29 \pm 10$ in patients; $39 \pm 9.8$ in controls; $p=0.004)$ and Symbol Digit Modalities Test $(50 \pm 7.8$ in patients; $57.5 \pm 8.4$ in controls; $p=0.006$ ) from the BRB. MFIS scores were significantly higher in patients compared to controls $(27.5 \pm 21$ in patients; $9.6 \pm 9.2$ in controls; $p=0.001)$.

\subsection{Subliminal and conscious priming effects}

In the priming experiment, we measured the ability of the subliminal number prime to influence the processing of the subsequent conscious target. We selected the delays where the prime was always processed unconsciously for both groups (SOA $16 \mathrm{~ms}$ and SOA $33 \mathrm{~ms}$ ) and those where the prime was always processed consciously (SOA $100 \mathrm{~ms}$ and SOA $150 \mathrm{~ms}$ ). We then performed an analysis of variance (ANOVA) on error rate and on mean RT with factors of group, prime-target relation (congruent repeated, congruent non-repeated, and incongruent) and type of process (conscious and unconscious).

The effect of prime-target relation on error rate and on RT could be decomposed into two distinct effects: repetition priming and congruity priming.

The reaction times (RT) analysis revealed that patients were slower than controls both for conscious processing $(613 \mathrm{~ms}$ versus $695 \mathrm{~ms}, F(1,42)=4.866, p=0.033)$ and for unconscious processing $(530 \mathrm{~ms}$ versus $630 \mathrm{~ms}, F(1,42)=10.581, p=0.002)$ (see Fig. 2). The repetition priming effect on RT was significant in all subjects both for conscious processing $(F(1,42)=25.862$, $p<0.001)$ and for unconscious processing $(F(1,42)=12.832$, $p<0.001)$.

The repetition priming effect on RT was significant for controls both for conscious processing $(F(1,21)=11.827, p=0.002)$ and for unconscious processing $(F(1,21)=7.988, p=0.01)$, as well as in patients $(F(1,21)=14.378, p=0.001$ for conscious processing; $F(1,21)=4.95, p=0.03$ for unconscious processing). There were no group $\times$ repetition priming inter-

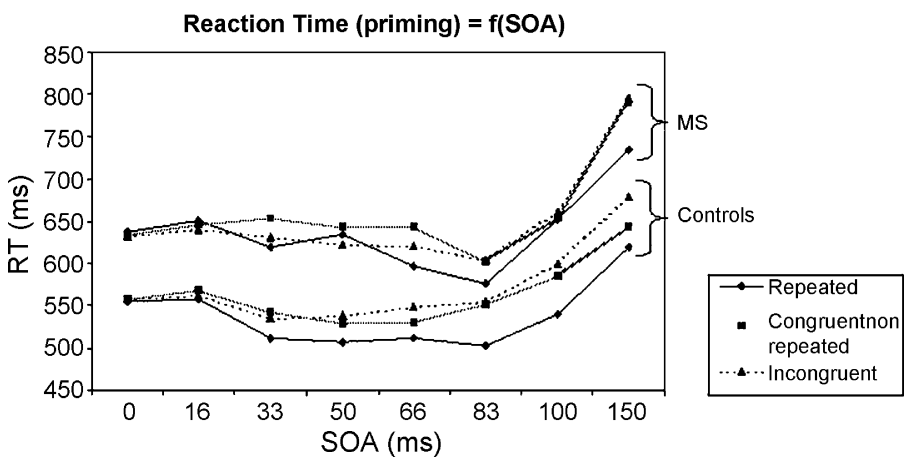

Fig. 2. Performances (reaction times) of MS patients and controls for the priming experiment. Trials are divided in three conditions defined by the prime-target relation: congruent repeated, congruent non-repeated and incongruent. The figure shows mean reaction times (RT) for each group, for each condition of prime-target relation and each SOA.

action neither for unconscious processing $(F(1,42)=0.275$, $p=0.603)$ nor for conscious processing $(F(1,42)=0.008$, $p=0.93)$.

The congruity priming effect on RT was significant in all subjects both for conscious processing $(F(1,42)=5.906, p=0.019)$ and for unconscious processing $(F(1,42)=6.686, p=0.013)$. The congruity priming effect on RT was significant in controls for the conscious processing $(F(1,21)=7.108, p=0.014)$ but was not significant for unconscious processing $(F(1,21)=1.956$, $p=0.177)$. In patients, congruity priming effect was not significant for conscious processing $(F(1,21)=0.329, p=0.572)$ but was significant for unconscious processing $(F(1,21)=4.8$, $p=0.04)$. There was no group $\times$ congruity priming interaction for unconscious processing $(F(1,42)=0.721, p=0.4)$ and only a trend for conscious processing $(F(1.42)=2.911, p=0.095)$.

By contrast, the error analysis revealed no significant priming effects. The error rate in target comparison to 5 was low and not significantly different between controls and patients, for the conscious processing $(4.46 \%$ errors for controls and $5.96 \%$ errors for patients, $F(1,42)=0.850, p=0.362$ ) as well as for the unconscious processing $(2.47 \%$ errors for controls and $4.28 \%$ errors for patients, $F(1,42)=1.880, p=0.178$ ).

The repetition priming effect on error rate was not significant, both when computed over all subjects both on conscious processing $(F(1,42)=1.224, p=0.275)$ and on unconscious processing $(F(1,42)=0.167, p=0.684)$. The repetition priming effect on error rate was not significant for controls neither on conscious processing $(F(1,21)=2.272, p=0.147)$ nor on unconscious processing $(F(1,21)=0.022, p=0.884)$, as well as in patients $(F(1,21)=0.012, p=0.912$ for conscious processing; $F(1,21)=0.492, p=0.491$ for unconscious processing). There was no group $\times$ repetition priming interaction neither for unconscious processing $(F(1,42)=0.374, p=0.544)$ nor for conscious processing $(F(1,42)=0.888, p=0.351)$.

Similarly, the congruity priming effect on error rate was not significant for all subjects both on conscious processing $(F(1,42)=0.675, p=0.146)$ and on unconscious processing $(F(1,42)=1.629, \quad p=0.209)$. The congruity priming effect on error rate was not significant for controls neither on conscious processing $(F(1,21)=1.129, p=0.3)$ nor on 
unconscious processing $(F(1,21)=0.023, p=0.879)$, as well as in patients $(F(1,21)=3.088, p=0.093$ for conscious processing; $F(1,21)=2.172, p=0.155$ for unconscious processing). There was no group $\times$ congruity priming interaction for unconscious processing $(F(1,42)=1.198, p=0.28)$. By contrast, there was a group $\times$ congruity priming interaction for conscious processing $(F(1,42)=4.196, p=0.047)$.

\subsection{Measuring the threshold for access to consciousness}

We studied the distributions of visibility scores in each group and for each SOA. In both groups, we observed a bimodal dis-
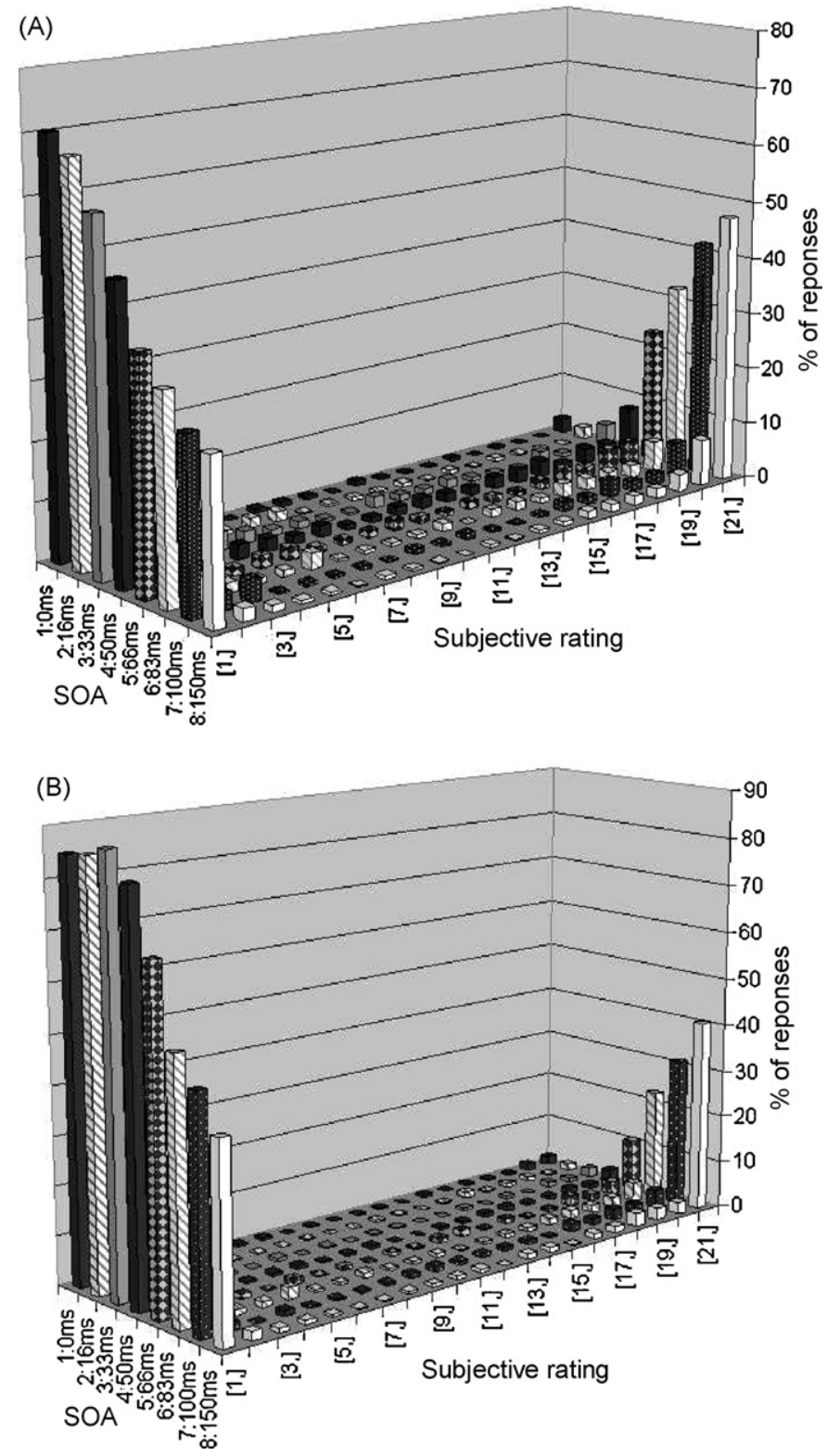

Fig. 3. Distribution of subjective visibility ratings for the masking experiment. Both in controls (A) and in patients (B), we observed a bimodal repartition of subjective visibility ratings, with a first set of responses close to maximal visibility (scale score $>17$ ) and a second set of responses peaking at zero visibility $($ score $<5)$. Responses between 5 and 17 were rare $(<10 \%)$. These bimodal distributions suggest that conscious visual perception is an 'all-or-none' process in both groups. tribution of scores (see Fig. 3), with a first set of responses close to maximal visibility (scale score $>17$ ) and a second set of responses peaking at zero visibility (scale score $<5$ ). The sum of these two sets of responses represented $91 \%$ of total responses in controls and $94 \%$ in patients without significant between-group differences (Mann-Whitney: $p=0.083$ ). Thus, in both groups, increasing delays did not lead to a progressive increase in subjective experience of prime visibility, but to a shift in the probability of reporting one of two discrete subjective states ("seen" or "not seen"). Based on this bimodal distribution, we arbitrarily defined "seen" trials as those whose visibility score was above the middle of the scale (Fig. 4). The proportion increased steadily with SOA, but at a slower rate in patients than in the controls. This pattern was evaluated with an ANOVA on the proportion of "seen trials", with factors of group and delay. The proportion of "seen" trials was significantly higher in controls than in patients $(F(1,25)=33.17 ; p<0.001)$. We also found a significant SOA effect $(F(7,173)=146.79 ; p<0.001)$ and a group $\times$ SOA interaction $(F(7,173)=10.06 ; p<0.001)$.

To analyse the objective measure of prime perception, we calculated in the two groups and for all subjects the percentage of correct responses in prime comparison to 5 for each SOA (Fig. 5). Those results were evaluated with an ANOVA with factors of group and SOA. The performance was significantly higher in controls than in patients $(76 \%$ against $62 \%$ correct; $F(1,25)=45.48 ; p<0.001)$. We found a significant SOA effect $(F(7,173)=81.05 ; p<0.001)$ and a group $\times$ SOA interaction $(F(7,173)=5.34 ; p<0.001)$. At each SOA, the controls outperformed the patients.

For five MS patients, objective and subjective thresholds could not be calculated because of a lack of conscious perception of the prime even at the longest SOA $(150 \mathrm{~ms})$. They were excluded from the analysis aiming to compare the threshold of access to consciousness between patients and controls. The thresholds were defined as the SOA for which the sigmoid curve

\section{Subjective threshold for access to consciousness}

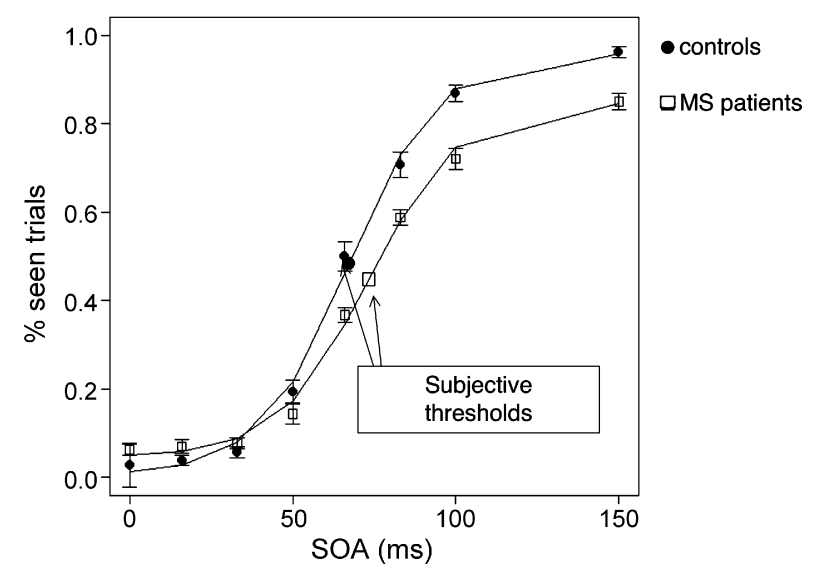

Fig. 4. Measures to determine the subjective threshold for access to consciousness. The figure displays the proportion of trials subjectively rated as "seen" as a function of SOA. The sigmoid curve fitting the data is represented as a continuous line. The mean subjective threshold was defined in each group as the SOA for which the sigmoid curve reached its inflexion point error. Bars represent the standard error. 


\section{Objective threshold for access to consciousness}

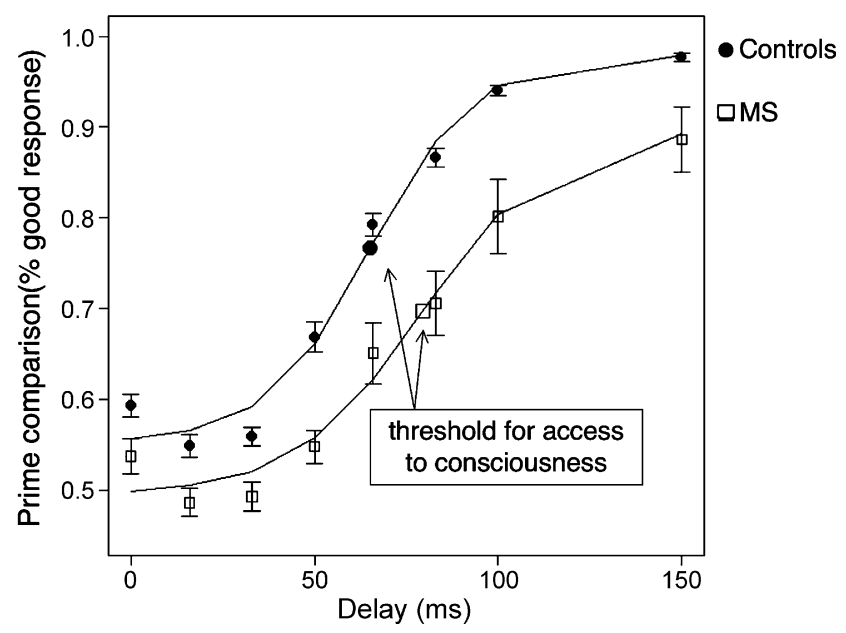

Fig. 5. Measures to determine the objective threshold for access to consciousness. The figure displays the percentage of correct responses in prime comparison to 5 as a function of delay (SOA). The sigmoid curve fitting the data is represented as a continuous line. The mean objective threshold was defined in each group as the SOA for which the sigmoid curve reached its inflexion point. Error bars represent the standard error.

fitted to the data reached its inflexion point (parameter $\alpha_{4}$, see Section 2 and Figs. 4 and 5).

The subjective threshold was significantly higher for patients $(76 \mathrm{~ms}$; S.D. $=14)$ compared to controls $(67 \mathrm{~ms}$; S.D. $=15.2)$ ( $p=0.012$; ANOVA with age and educational level as confounding covariates).

The objective threshold was significantly higher for patients (77 ms; S.D. = 16) compared to controls (63 ms; S.D. =15) $(p=0.0004$; ANOVA with age and educational level as confounding covariates).

The objective and subjective thresholds were highly correlated within the controls (corrected $\rho=0.93, p<0.001$; Spearman rank test) but also within the patients (corrected $\rho=0.89, p<0.001 ;$ Spearman rank test).

In patients, no correlation were observed between thresholds (objective and subjective) and MFIS, Word List Generation task and SDMT scores ( $p>0.3$; Spearman rank test).

\section{Discussion}

\subsection{Visual backward masking task}

The visual backward masking paradigm used in the present study differs from the one used previously in Sergent et al. (Sergent \& Dehaene, 2004). Here, a form of metacontrast masking was used whereas a pattern masking was used in Sergent et al. Our explanation concerning the differences in results obtained between the two studies in controls, is that the low level effect of pattern masking modifies linearly the subjective report for perception whereas in the present masking paradigm, the main masking effect concerns central attentional mechanism and thus leads to non-linear perceptual discontinuity.

The differences of delivery modalities between the two paradigms used in the present study (blocked in the priming ver- sus randomly in the masking experiment) may induce a slight shift in the value of the threshold access to consciousness. In fact, attentional focusing during blocks relative to randomly delivered priming may have lead to a decrease in masking threshold, in comparison with the threshold measured in masking experiment where SOA were randomly presented. However, it seems that an increase of the threshold in priming experiment in comparison with the masking experiment is more likely because in the former, subjects have to focus their attention on the mask (the target number belongs to the mask) and neglect the prime whereas in the latter, they neglect the mask and focus on the prime. Anyway, these attentional factors leading potentially to a shift in conscious threshold of the prime between both experiments could differ between patients and controls. This confounding effect was accounted for the analyse of unconscious versus conscious priming, by removing the delays around the threshold values determined by the masking experiment (we kept 16 and $33 \mathrm{~ms}$ for unconscious processing, and 100 and $150 \mathrm{~ms}$ for conscious processing).

\subsection{Subliminal processing and conscious access in multiple sclerosis}

In the present study we demonstrated that the threshold for access to consciousness of masked stimuli is higher in early MS patients compared to controls, whereas subliminal priming (indicating fast feed-forward processing of these masked stimuli) is preserved. To our knowledge, this is the second experiment in cognitive psychology studying visual backward masking performance in MS. The previous study found that some patients with optic neuritis had a poor performance in a visual masking test (Marx et al., 1984). The present study is the first to separate deficits imputable to the late stages of conscious perception and to early low-level visual processing. The results support our hypothesis that the elevated masking threshold in MS is due to dysfunction primarily at late stages of conscious perception.

We tentatively propose that the mechanism for the deficit is the diffuse white matter damage observed in early MS, which affects cortico-cortical recurrent activity necessary for access to consciousness. An alternative explanation of the elevated masking threshold would be an early visual dysfunction at the level of the optic nerve or of the early visual pathways which conduct information to primary and secondary visual areas. Two reasons suggest that this low-level mechanism can be ruled out here. First, the absence of history or signs of optic neuritis was carefully ascertained. Moreover, a putative infra-clinical optic neuritis responsible for slower conduction in the optic nerves would not account for the present result. Indeed, the measure of the consciousness threshold was based on the time interval between prime and target. Therefore, it should not be affected by an additive optic nerve delay.

This argument supposes that a putative neuritis would only affect the delay but not the variability of cortical arrival times, which is a debatable assumption. However, a second, more crucial argument against putative low-level visual deficits is the finding of a normal subliminal priming effect. Behavioural and neuroimaging studies of subliminal priming suggest that 
masked invisible stimuli are processed extensively, including visual recognition, but also lexical and even semantic levels (Dehaene, 2004; Dehaene et al., 2004, 2001; Greenwald, Draine, \& Abrams, 1996; Naccache \& Dehaene, 2001). The preservation of non-conscious priming in MS suggests that the fast feed-forward processing stages supporting these priming effects must be largely intact, including conduction from optic nerve to visual cortex, but also object recognition, numerical comparison and automatic response programming. The diffuse white matter injuries found in early MS seem not to affect the efficiency of these non-conscious processes, presumably relying on localized neuronal networks. The situation may of course be different in patients with long-lasting MS, where the importance of diffuse neuronal loss may induce significant alteration of these encapsulated processes.

As previously described, the "conscious neuronal workspace" theory emphasizes the role of long distance connections necessary for the emergence of a self-amplified reverberant neuronal assembly which connects together distant brain areas, particularly prefrontal, cingulate and parietal cortices, and which is associated with conscious reportability (Dehaene et al., 1998, 2003). Diffuse demyelinating processes in patients may disturb the connectivity between distant cortical areas (Au Duong, Audoin et al., 2005; Au Duong, Boulanouar et al., 2005), and thus provide a mechanism for the elevated threshold for access to consciousness. Indeed, detailed simulations of thalamo-cortical networks indicate that the threshold for ignition of a broad cell assembly and reverberation of activation across distant cortical regions depends sharply on the strength and speed of recurrent connections (Dehaene \& Changeux, 2005). At the early stage of MS, numerous studies have demonstrated diffuse white matter injury, characterized by macroscopic but also microscopic tissue damage (Miller, Thompson, \& Filippi, 2003). In particular diffuse injury has recently been described in the corpus callosum - a structure composed solely of long inter-hemispheric associative fibers in patients with relapsing remitting MS (Audoin et al., 2007; Pelletier et al., 2001; Ranjeva et al., 2003). We previously demonstrated, using voxel-based analysis of brain magnetization transfer ratio (MTR) maps, a sensitive technique allowing to evidence subtle tissue injury outside $\mathrm{T}_{2}$ visible lesions, that in patients with early MS, long range white matter tracts were significantly injured, without diffuse injury in the cortex (Ranjeva et al., 2004). Moreover, MR studies performed in patients at the early stage of the disease have demonstrated that tissue injury was mostly characterized by demyelinating processes, without significant axonal loss (Audoin et al., 2007; Fernando et al., 2004; Ranjeva et al., 2003). Connectivity disturbance may also be responsible for the elevated threshold for access to consciousness evidenced in patients with schizophrenia (Delcul et al., 2006; K. Friston, 2005; K.J. Friston, 1998; Whalley et al., 2005), although the detailed neurobiological mechanism might be partially different, perhaps involving abnormal synaptic plasticity (Frankle et al., 2003).

In the current study, we found that in controls as well as in patients the responses obtained on the subjective visibility scale showed a bimodal distribution. This suggests that conscious per- ception is determined by a stochastic 'all-or-none' dynamical transition as predicted by the conscious neuronal workspace theory (Dehaene et al., 1998, 2003). This characteristic of conscious access appears similar in patients compared to controls, although the threshold for access to consciousness is delayed in patients. Moreover, the tight correlation between objective and subjective thresholds within each subject and in both groups indicates that patients have a reliable introspection about their vision. This also suggests that the variability found in the masking thresholds was not noise but could be considered as a genuine inter-individual difference between subjects. These findings seem to indicate that access to consciousness follows a comparable process in patients and in controls but with a significant longer delay in patients.

The present study demonstrates, for the first time, that patients with early MS have normal subliminal processing but delayed access to consciousness compared to healthy controls. These results corroborate previous studies performed in patients with relapsing remitting MS, which have clearly demonstrated that cognitive impairment is frequent in MS and is mainly represented by a deficiency in controlled conscious information processing (Achiron \& Barak, 2003; Deloire et al., 2005; Feuillet et al., 2007). In the future, diffusion tensor imaging, functional magnetic resonance imaging, and event-related potentials could be used to specify the topography and the precise chronometry of brain activity evoked by masked stimuli, and to directly correlate deficits of conscious access with anatomical white-matter impairments.

\section{Conflict of interest}

Any authors declare that they have no conflict of interest relative to this research.

\section{Acknowledgements}

This work was supported by CNRS, INSERM and ARSEP.

\section{References}

Achiron, A., \& Barak, Y. (2003). Cognitive impairment in probable multiple sclerosis. Journal of Neurology, Neurosurgery, and Psychiatry, 74(4), 443-446.

Au Duong, M. V., Audoin, B., Boulanouar, K., Ibarrola, D., Malikova, I., Confort-Gouny, S., et al. (2005). Altered functional connectivity related to white matter changes inside the working memory network at the very early stage of MS. Journal of Cerebral Blood Flow and Metabolism, 25(10), $1245-1253$.

Au Duong, M. V., Boulanouar, K., Audoin, B., Treseras, S., Ibarrola, D., Malikova, I., et al. (2005). Modulation of effective connectivity inside the working memory network in patients at the earliest stage of multiple sclerosis. Neuroimage, 24(2), 533-538.

Audoin, B., Au Duong, M. V., Ranjeva, J. P., Ibarrola, D., Malikova, I., ConfortGouny, S., et al. (2005). Magnetic resonance study of the influence of tissue damage and cortical reorganization on PASAT performance at the earliest stage of multiple sclerosis. Human Brain Mapping, 24(3), 216-228.

Audoin, B., Ibarrola, D., Malikova, I., Soulier, E., Confort-Gouny, S., Au Duong, M. V., et al. (2007). Onset and underpinnings of white matter atrophy at the very early stage of multiple sclerosis-a two-year longitudinal MRI/MRSI study of corpus callosum. Multiple Sclerosis, 13(1), 41-51. 
Beck, D. M., Rees, G., Frith, C. D., \& Lavie, N. (2001). Neural correlates of change detection and change blindness. Nature Neuroscience, 4(6), 645650.

Breitmeyer, B. G. (1984). Visual masking: an integrative approach. Oxford: Oxford University Press.

Breitmeyer, B. G., \& Ogmen, H. (2000). Recent models and findings in visual backward masking: a comparison, review, and update. Perception and Psychophysics, 62(8), 1572-1595.

Cader, S., Cifelli, A., Abu-Omar, Y., Palace, J., \& Matthews, P. M. (2006). Reduced brain functional reserve and altered functional connectivity in patients with multiple sclerosis. Brain, 129(Pt 2), 527-537.

Callanan, M. M., Logsdail, S. J., Ron, M. A., \& Warrington, E. K. (1989). Cognitive impairment in patients with clinically isolated lesions of the type seen in multiple sclerosis. A psychometric and MRI study. Brain, 112(Pt 2), 361-374.

Dehaene, S. (2004). The neural basis of subliminal priming. In N. Kanwisher \& J. Duncan (Eds.), Functional neuroimaging of visual cognition (attention and performance series). New York: Oxford University Press.

Dehaene, S., \& Changeux, J. P. (2004). Neural mechanisms for access to consciousness. In M. Gazzaniga (Ed.), The cognitive neurosciences (3rd ed., pp. 1145-1157). New York: Bradford Book.

Dehaene, S., \& Changeux, J. P. (2005). Ongoing spontaneous activity controls access to consciousness: a neuronal model for inattentional blindness. PLoS Biology, 3(5), e141.

Dehaene, S., Changeux, J. P., Naccache, L., Sackur, J., \& Sergent, C. (2006). Conscious, preconscious, and subliminal processing: a testable taxonomy. Trends in Cognition Science, 10(5), 204-211.

Dehaene, S., Jobert, A., Naccache, L., Ciuciu, P., Poline, J. B., Le Bihan, D., et al. (2004). Letter binding and invariant recognition of masked words: behavioral and neuroimaging evidence. Psychological Science, 15(5), $307-$ 313.

Dehaene, S., Kerszberg, M., \& Changeux, J. P. (1998). A neuronal model of a global workspace in effortful cognitive tasks. Proceedings of the National Academic Science of United States of America, 95(24), 1452914534.

Dehaene, S., \& Naccache, L. (2001). Towards a cognitive neuroscience of consciousness: basic evidence and a workspace framework. Cognition, 79(1-2), $1-37$.

Dehaene, S., Naccache, L., Cohen, L., Bihan, D. L., Mangin, J. F., Poline, J. B., et al. (2001). Cerebral mechanisms of word masking and unconscious repetition priming. Nature Neuroscience, 4(7), 752-758.

Dehaene, S., Sergent, C., \& Changeux, J. P. (2003). A neuronal network model linking subjective reports and objective physiological data during conscious perception. Proceedings of the National Academic Science of United States of America, 100(14), 8520-8525.

Delcul, A., Dehaene, S., \& Leboyer, M. (2006). Preserved subliminal processing and impaired conscious access in Schizophrenia. Archives of General Psychiatry, 63(12), 1313-1323.

Deloire, M. S., Salort, E., Bonnet, M., Arimone, Y., Boudineau, M., Amieva, H., et al. (2005). Cognitive impairment as marker of diffuse brain abnormalities in early relapsing remitting multiple sclerosis. Journal of Neurology, Neurosurgery, and Psychiatry, 76(4), 519-526.

Di Lollo, V., Enns, J. T., \& Rensink, R. A. (2000). Competition for consciousness among visual events: the psychophysics of reentrant visual processes. Journal of Experimental Psychology General, 129(4), 481-507.

Fernando, K. T., McLean, M. A., Chard, D. T., MacManus, D. G., Dalton, C. M., Miszkiel, K. A., et al. (2004). Elevated white matter myo-inositol in clinically isolated syndromes suggestive of multiple sclerosis. Brain, $127(\mathrm{Pt}$ 6), 1361-1369.

Feuillet, L., Reuter, F., Audoin, B., Malikova, I., Barrau, K., Ali Cherif, A., et al. (2007). Early cognitive impairment in patients with clinically isolated syndrome suggestive of multiple sclerosis. Multiple Sclerosis, 13(1), 124-127.

Fisk, J. D., Ritvo, P. G., Ross, L., Haase, D. A., Marrie, T. J., \& Schlech, W. F. (1994). Measuring the functional impact of fatigue: initial validation of the fatigue impact scale. Clinical Infectious Diseases, 18(Suppl. 1), S79-S83.

Frankle, W. G., Lerma, J., \& Laruelle, M. (2003). The synaptic hypothesis of schizophrenia. Neuron, 39(2), 205-216.
Friston, K. (2005). Disconnection and cognitive dysmetria in schizophrenia. American Journal of Psychiatry, 162(3), 429-432.

Friston, K. J. (1998). The disconnection hypothesis. Schizophrenia Research, $30(2), 115-125$.

Greenwald, A. G., Draine, S. C., \& Abrams, R. L. (1996). Three cognitive markers of unconscious semantic activation. Science, 273(5282), 16991702.

Gross, J., Schmitz, F., Schnitzler, I., Kessler, K., Shapiro, K., Hommel, B., et al. (2004). Modulation of long-range neural synchrony reflects temporal limitations of visual attention in humans. Proceedings of the National Academic Science of United States of America, 101(35), 13050 13055.

Haynes, J. D., Driver, J., \& Rees, G. (2005). Visibility reflects dynamic changes of effective connectivity between V1 and fusiform cortex. Neuron, 46(5), $811-821$.

Heaton, R. K., Nelson, L. M., Thompson, D. S., Burks, J. S., \& Franklin, G. M. (1985). Neuropsychological findings in relapsing-remitting and chronic-progressive multiple sclerosis. Journal of Consulting and Clinical Psychology, 53(1), 103-110.

Kurtzke, J. F. (1983). Rating neurologic impairment in multiple sclerosis: an expanded disability status scale (EDSS). Neurology, 33(11), 1444 1452.

Lamme, V. A. (2003). Recurrent corticocortical interactions in neural disease. Archives of Neurology, 60(2), 178-184.

Lamme, V. A. (2006). Towards a true neural stance on consciousness. Trends in Cognition Science, 10(11), 494-501.

Lamme, V. A., \& Roelfsema, P. R. (2000). The distinct modes of vision offered by feedforward and recurrent processing. Trends in Neuroscience, 23(11), 571-579.

Lau, H. C., \& Passingham, R. E. (2006). Relative blindsight in normal observers and the neural correlate of visual consciousness. Proceedings of the National Academic Science of United States of America, 103(49), 1876318768.

Lyon-Caen, O., Jouvent, R., Hauser, S., Chaunu, M. P., Benoit, N., Widlocher, D., et al. (1986). Cognitive function in recent-onset demyelinating diseases. Archives of Neurology, 43(11), 1138-1141.

Marois, R., Chun, M. M., \& Gore, J. C. (2000). Neural correlates of the attentional blink. Neuron, 28(1), 299-308.

Marois, R., Yi, D. J., \& Chun, M. M. (2004). The neural fate of consciously perceived and missed events in the attentional blink. Neuron, 41(3), 465472.

Marx, M. S., May, J. G., Reed, J. L., Matteson, H. H., van Dyk, H. J., \& Jayaraman, A. (1984). Spatio-temporal processing in multiple sclerosis. Docmenta Ophthalmologica, 56(3), 243-264.

Miller, D. H., Thompson, A. J., \& Filippi, M. (2003). Magnetic resonance studies of abnormalities in the normal appearing white matter and grey matter in multiple sclerosis. Journal of Neurology, 250(12), 14071419.

Naccache, L., \& Dehaene, S. (2001). Unconscious semantic priming extends to novel unseen stimuli. Cognition, 80(3), 215-229.

Pelletier, J., Suchet, L., Witjas, T., Habib, M., Guttmann, C. R., Salamon, G., et al. (2001). A longitudinal study of callosal atrophy and interhemispheric dysfunction in relapsing-remitting multiple sclerosis. Archives of Neurology, 58(1), 105-111.

Peyser, J. M., Edwards, K. R., Poser, C. M., \& Filskov, S. B. (1980). Cognitive function in patients with multiple sclerosis. Archives of Neurology, 37(9), 577-579.

Ranjeva, J., Audoin, B., Au Duong, M. V., Ibarrola, D., Malikova, I., ConfortGouny, S., et al. (2004). Statistical mapping analysis of brain MTR: a sensitive method to study relationship between tissue damage and functional status of patients at the earliest stage of multiple sclerosis. American Journal of Neuroradiology, 26(1), 119-127.

Ranjeva, J., Pelletier, J., Confort-Gouny, S., Ibarrola, D., Audoin, B., Le Fur, Y., et al. (2003). MRI/MRS of corpus callosum in patients with clinically isolated syndrome suggestive of multiple sclerosis. Multiple Sclerosis, 9(6), 554-565.

Rao, S. (1990). A manual for the Brief Repeatable Battery of Neuropsychological Tests in multiple sclerosis. Milwaukee: Medical College of Wisconsin. 
Rao, S. M., Leo, G. J., Bernardin, L., \& Unverzagt, F. (1991). Cognitive dysfunction in multiple sclerosis. I. Frequency, patterns, and prediction. Neurology, 41(5), 685-691.

Sergent, C., Baillet, S., \& Dehaene, S. (2005). Timing of the brain events underlying access to consciousness during the attentional blink. Nature Neuroscience, 8(10), 1391-1400.
Sergent, C., \& Dehaene, S. (2004). Is consciousness a gradual phenomenon? Evidence for an all-or-none bifurcation during the attentional blink. Psychological Science, 15(11), 720-728.

Whalley, H. C., Simonotto, E., Marshall, I., Owens, D. G., Goddard, N. H., Johnstone, E. C., et al. (2005). Functional disconnectivity in subjects at high genetic risk of schizophrenia. Brain, 128(Pt 9), 2097-2108. 